\title{
Minimizing the risk of childbirth-induced pelvic floor dysfunctions in the developing word: "preventive" urogynecology
}

\author{
Diaa E. E. Rizk
}

Received: 11 March 2009 /Accepted: 31 March 2009/Published online: 28 April 2009

(C) The International Urogynecological Association 2009

\begin{abstract}
"The complete protection of the perineum has undoubtedly remained a weak spot in our art" Ferdinand A.M.F. von Ritgen ${ }^{1}, 1855$.
\end{abstract}

Normal childbirth inevitably damages the pelvic floor, especially when non-evidence-based and potentially harmful obstetric practices are used during management of labor. Direct injury to the pelvic floor muscles significantly contributes to weakness of supportive function and development of support-related pelvic floor dysfunctions such as pelvic organ prolapse, urodynamic stress urinary incontinence (USI) and fecal incontinence (FI). Spontaneous perineal lacerations also commonly occur at vaginal delivery, particularly in primiparous women, causing either occult or recognized anal sphincter disruption with subsequent FI in 50\% of cases [1]. Obstetric vesico- and rectovaginal fistulae represent the final "kiss of death" in a protracted labor scenario with ischemic necrosis and sloughing of the vaginal and bladder or rectal tissues interposed between the pelvic bones and presenting fetal part. Since the objective here is to focus on the earlier stages of birth trauma when recovery of pelvic floor supportive function is theoretically possible before loss of

\footnotetext{
$\overline{{ }^{1} \text { German obstetrician (1787-1867) who described the Ritgen }}$ maneuver: delivery of a child's head by pressure on the perineum while controlling the speed of delivery by pressure with the other hand on the head.
}

D. E. E. Rizk $(\bowtie)$

Department of Obstetrics and Gynecology, Faculty of Medicine,

Ain Shams University,

Abbasia,

Cairo 22367, Egypt

e-mail: rizk.diaa@gmail.com structural integrity of pelvic organs, obstetric fistulae will not be discussed further.

Episiotomy has been traditionally performed by obstetric care providers to protect against direct trauma to the pelvic floor muscles during parturition. However, the value of this procedure is increasingly questioned by the urogynecologic community because of the associated postoperative morbidity in the absence of clearly defined evidence for efficacy [2]. In fact, episiotomy, particularly if median, is paradoxically associated with more anal sphincter injury and decreased perineal muscle strength and performance during the postpartum period than spontaneous perineal lacerations, because of greater tissue disruption $[1,2]$. Moreover, the optimum ratio between the length of episiotomy and the length of perineum that can reliably predict minimal perineal damage is not known [1]. Despite the availability of this information, episiotomy, particularly the routine procedure, is performed more frequently in developing countries than in the West [3].

Over the past two decades, neurophysiological and experimental research has revealed that vaginal delivery damages the pelvic floor muscles through mechanisms unrelated to traumatic disruption [4-6]. During the second stage of labor, the pudendal nerve is compressed by the fetal head against the ischial spines, causing entrapment neuropathy and partial denervation of the striated pelvic floor muscles [4]. More importantly, in labor simulation computer and animal models, the fetal head and biomechanical labor forces cause direct stretch and compression injuries to the pelvic floor muscles during delivery that contributes to progressive deterioration in muscle function. Both muscle injuries were found to develop at a lower tissue elasticity threshold than that observed in normal 
parturition $[5,6]$. The likelihood of indirect neural and muscular injuries is obviously increased when labor dystocia is not detected and treated promptly, or when aggressive maneuvers are used to expedite delivery, such as forcible manual compression of the uterus or dilatation of the vagina and excessive traction by the obstetric forceps or ventouse. Regrettably, these obstetric interventions are still "endemic" in the developing world, mainly in rural communities and remote geographical areas [3].

At present, there are sufficient epidemiological data to suggest that pregnancy also has a detrimental effect on pelvic floor supportive function. In the seminal EPINCONT study, there was a higher risk of developing USI amongst women who had had elective cesarean delivery than in nulliparous women, representing a net effect of pregnancy itself [7]. Although the exact mechanisms during gestation remain obscure, increased urine production, degenerative changes in the autonomic innervation of the lower urinary tract, mechanical pressure exerted by the gravid uterus and elevated elastolytic activity with softening of the collagenous supports of the bladder neck and proximal urethra may be responsible [8]. Repeated pregnancy will conceivably impart a negative cumulative influence on the pelvic floor support similar to other pregnancy-induced disorders like diabetes mellitus and hypertension. Although several national birth control initiatives had been used in most developing countries since the 1960s, the grand multiparity and total fertility rates have been constantly rising in the new millennium [9].

There is strong contemporary evidence that pelvic floor muscle training (PFMT) during pregnancy or the postnatal period can prevent USI in late pregnancy or postpartum USI and FI, respectively, with a greater treatment effect following more intensive regimes. In a recent systematic review, pregnant women without prior USI who were randomized to antenatal PFMT were 56\% and 30\% less likely than women randomized to no PFMT or usual antenatal care to report USI in late pregnancy and up to 6 months postpartum, respectively [10]. Postnatal women with persistent USI 3 months after delivery and who received PFMT were $20 \%$ less likely than women who did not receive treatment or received usual postnatal care to report USI 12 months after delivery. Women with postnatal FI who received PFMT were also about half as likely to report FI 12 months after delivery. PFMT, nevertheless, is rarely practiced during pregnancy or after delivery in most developing countries because of modest appreciation of its prophylactic value and inadequate human and physical resources.

In view of the hitherto increased risk of support-related pelvic floor dysfunctions following pregnancy and childbirth in low-resource and less developed countries, a growing demand for curative urogynecologic services is anticipated. The need may be higher than the actuarial reported prevalence of these dysfunctions, since the majority of women in the developing world do not seek health care [11]. Scientific reports and social monographs also consistently point to the persistence of the inverse health care law amongst women from these countries because of inequities in health care access, utilization and delivery - the availability of good-quality health care, including urogynecologic care, is inversely related to the women who need it. Although we now live in a world where subspecialization in obstetrics and gynecology is becoming ipso facto and leading to a more endoscopic vision of female pelvic floor health, this topic has a wider definition than the standard accepted terminology-appropriate urinary and fecal control, integrity of pelvic organ support and normal sexual function. In essence, pelvic floor health in women also incorporates the drive towards safer and less traumatic childbirth and hence, improvement in practice, management and conduct of labor and delivery [12]. The most pragmatic solution to restore the demand/ supply equilibrium in developing countries may be to reinforce this preventive perspective of urogynecologic practice and déjà vu the obstetric component of general training. The goal is to transcend across this novel and artificial subspecialty boundary and pursue the timehonored public health idiom, "prevention is better than cure".

The principal assignment of "preventive urogynecologists" is thus to look towards their twin obstetrics discipline and affiliated maternity units in order to raise awareness about childbirth-induced pelvic floor dysfunctions and disseminate information about the safe practices in labor. Although it is often harder to do "nothing" than do "something" for parturients, reproductive health literature is now freely available from the World Health Organization electronic library on an annual basis [3]. This can provide the birth attendants from developing countries with "fingeron-the-pulse" capability to ensure that labor guidelines remain updated and based on the best available evidence. In particular, performing routine versus selective episiotomy should be regarded as the last and most tragic monument to a myopic clinical philosophy of a bygone era to protect the pelvic floor. The bona fide adverse consequences of pregnancy on pelvic floor support should be highlighted to family planning physicians. The benefits of routine PFMT during pregnancy and after delivery should be communicated to maternal care providers, especially when targeted to specific groups like primiparous women and those who had USI in early pregnancy, prolonged labor or forceps/ventouse delivery. Capacity building of a cadre of career champions in pelvic floor physiotherapy backed up with modern equipment should be brought to the attention of health policy makers and other stakeholders as an urgent manpower and funding priority. Preventive urogynecolo- 
gists should always participate in grand rounds and morbidity reviews of near-miss or extensive pelvic floor injuries sustained during childbirth. Regular urogynecologic input can provide educational feedback to maternity staff, assist in identification and timely referral of potential complications, and preempt patient complaints and public criticism. These risk management and audit activities should not, however, provoke superfluous intradisciplinary sensitivity or offer an opening for the bureaucratic and regulatory instincts of administrators or other professional bodies.

Preventive urogynecologists of the developing world should assume the primary responsibility in explaining the risk of pelvic floor dysfunctions associated with pregnancy and vaginal delivery, thus improving the health literacy of their underprivileged pregnant population $[11,12]$. Information aids include print materials, videos and audioguided workbooks that can be used during counseling and preferably at the first antenatal visit [13]. These aids can be very useful for preference-sensitive health decisions related to pelvic floor dysfunctions like planned family size and mode of delivery where the woman's own preferences for risks and benefits should dictate her personal choice. There is an important influence of family, kin and friends on the woman's health decision, however, in most developing countries. Therein, the individual is part of an informal reciprocal system of network exchange, which is based on trust, has social consequences and entails duties and responsibilities for both sides. Other societal variables to consider are patriarchy (male dominance), cultural importance of childbearing and normal birth, religious values and perception of health role [12].

The outcome of preventive urogynecology endeavors in developing countries can be significantly improved if the clientele is actively involved. Women can contribute by understanding the mechanisms of pregnancy- and childbirth-induced injuries, self-managing their reproductive and birthing choices, complying with prophylactic measures before and after delivery and selecting appropriate treatment options for ante- and post-partum pelvic floor dysfunctions. Preventive urogynecologists from developing countries should, therefore, recognize and support par coeur the tête-à-tête physician-patient partnership model founded on skillful communication, knowledge transfer, information sharing, coaching and question prompts for patients and interactive dialogue [11]. Finally, government strategies and community outreach programs that promote women's education, emancipation, reproductive rights and engagement in childbirth-induced pelvic floor dysfunctions should be a fundamental plank of the preventive urogynecologic agenda in the developing world.
In conclusion, the mission of preventive urogynecologists in the developing word can be perfectly described by a verse in the poem by John Webster, The Duchess of Malfi (c. 1612):

"Past sorrows, let us moderately lament them, For those to come, seek wisely to prevent them" [14].

\section{Conflicts of interest None.}

\section{References}

1. Rizk DEE, Abadir MN, Thomas LB, Abu-Zidan F (2005) Determinants of the length of episiotomy or spontaneous posterior perineal lacerations during vaginal birth. Int Urogynecol $\mathrm{J}$ 16:395-400

2. Fritel X, Schaal JP, Fauconnier A, Bertrand V, Levet C, Pigné A (2008) Pelvic floor disorders 4 years after first delivery: a comparative study of restrictive versus systematic episiotomy. BJOG 1152:247-252

3. The WHO Reproductive Health Library: Pregnancy and Childbirth, Care during childbirth. http://www.who.int/rhl. Accessed 1st March, 2009

4. Snooks SJ, Swash M, Mathers SE, Henry MM (1990) Effect of vaginal delivery on the pelvic floor: a 5-year follow-up. Br J Surg 77:1358-1360

5. Lien KC, Mooney B, DeLancey JOL, Ashton-Miller JA (2004) Levator ani muscle stretch induced by simulated vaginal Birth. Obstet Gynecol 103:31-40

6. Pan HQ, Kerns JM, Lin DL, Liu S, Esparza N, Damaser MS (2007) Increased duration of simulated childbirth injuries results in increased time to recovery. Am J Physiol Regul Integr Comp Physiol 292:R1738-R1744

7. Rortveit G, Daltveit AK, Hannestad YS, Hunskaar S, Norwegian EPINCONT Study (2003) Urinary incontinence after vaginal delivery or cesarean section. N Engl J Med 348:900-907

8. Fitzgerald MP, Graziano S (2007) Anatomic and functional changes of the lower urinary tract during pregnancy. Urol Clin North Am 34:7-12

9. 2008 World Population Data Sheet (2008). The Population Reference Bureau. www.prb.org.. Accessed 1st March, 2009

10. Hay-Smith J, Mørkved S, Fairbrother KA, Herbison GP (2008) Pelvic floor muscle training for prevention and treatment of urinary and faecal incontinence in antenatal and postnatal women. Cochrane Database Syst Rev 8:4

11. Rizk DEE (2008) Ethnic differences in women's knowledge level and other barriers to care seeking and the true incidence and/or prevalence rate of female pelvic floor disorders. Int Urogynecol J 19:1587-1588

12. Rizk DEE, El-Safty MM (2006) Female pelvic floor dysfunction in the Middle East: a tale of three factors: culture, religion and socialization of health role stereotypes. Int Urogynecol J 17:436438

13. O'Connor AM, France Legare F, Stacey D (2003) Risk communication in practice: the contribution of decision aids. BMJ 327:736-740

14. Emery AEH, Emery MLH (2007) Mother and child care in art. Royal Society of Medicine, London, $\mathrm{p}$ ix 